\title{
Strömungswiderstand des Gerätes spielt eine Rolle
}

Die Inhalationstherapie stellt je nach Gerätetyp unterschiedliche Anforderungen an den Patienten. Bei Pulverinhalatoren spielt dabei nicht nur der Atemfluss bei der Hubauslösung zur Erzeugung eines Aerosols eine Rolle. Auch der innere Gerätewiderstand spielt eine für den Patienten relevante Rolle, berichtete Dr. Peter Haidl, Schmallenberg, in München ${ }^{1}$.

In Zusammenarbeit mit dem Fraunhofer Institut für Toxikologie und Experimentelle Medizin in Hannover untersuchte Haidl die Druckabnahme in Abhängigkeit von der Atemflussrate bei 14 handelsüblichen Geräten (Dosieraerosole und Pulverinhalatoren $)^{2}$. Dazu wurde mit einem elektronischen Druckluftsensor der jeweilige Druckluftabfall ermittelt. Die Inhalatoren zeigten in den Untersuchungen deutliche Unterschiede aufgrund des inneren Widerstands, der sich je nach Flussrate unterschiedlich stark auswirkte. Den geringsten Gerätewiderstand wiesen die Dosieraerosole auf, den höchsten der Pulverinhalator Handihaler ${ }^{\circledR}$.

\section{Atemleistung in Abhängigkeit vom Gerätewiderstand}

Der Gerätewiderstand stellt über den inspiratorischen Mindestatemfluss hinaus, der bei Dosieraerosolen bei ca. $101 / \mathrm{min}$, bei Pulverinhalatoren bei bis $\mathrm{zu} 401 / \mathrm{min}$ oder sogar $501 /$ min liegen kann, eine zusätzliche Anforderung an den Patienten.

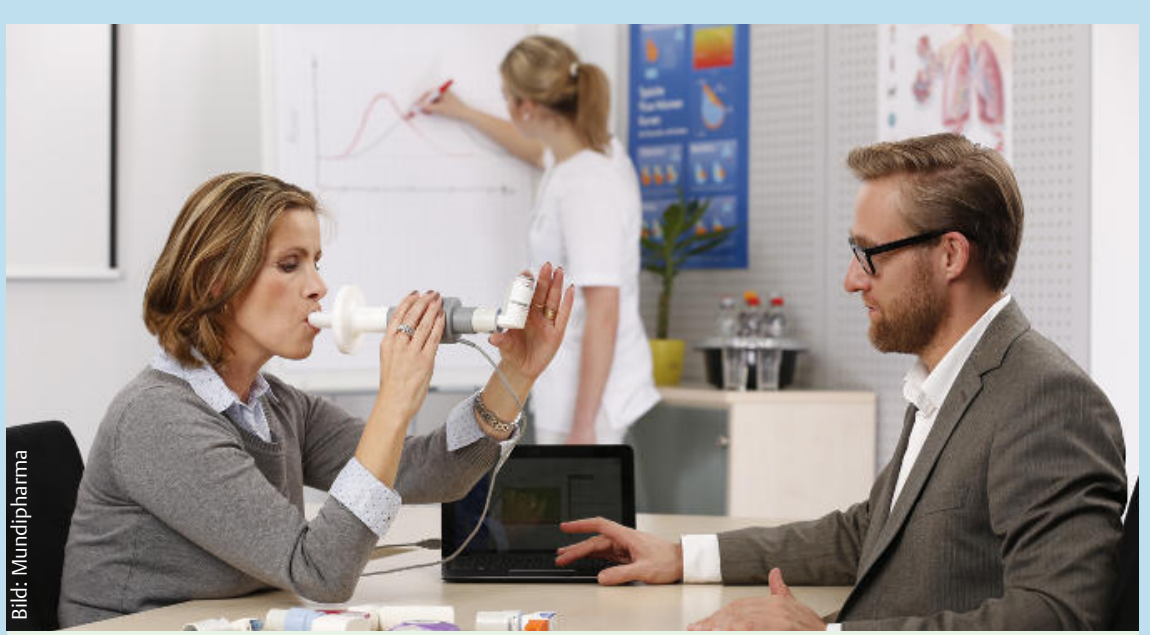

Der Inhalations-Coach misst verschiedene Parameter des Inhalationsmanövers wie Atemfluss, Flussbeschleunigung, Inhalationsdauer und inspiratorisches Volumen, deckt Koordinationsfehler auf und visualisiert die Ergebnisse.
„Aus den gemessenen Gerätewiderstandswerten und den Mindestflüssen lässt sich die Atemleistung errechnen, die ein Patient zum korrekten Inhalieren benötigt“, erklärte Haidl. So wird nach seinen Untersuchungen für eine optimale inspiratorische Flussrate beim Handihaler ${ }^{\circledR}$ schon eine Kraft von 1,3 Watt, beim Turbohaler $^{\circledR}$ gar von etwa 4,3 Watt benötigt. Gesunde schafften 15 - 20 Watt, erläuterte Haidl, bei Obstruktion kämen Patienten aber oft nur auf 1 - 2 Watt.

Bei treibgasgestützten Dosieraerosolen (z.B. Fluticason plus Formoterol ${ }^{3}$ ) ist dagegen auch nach den aktuellen Studienergebnissen eine sehr geringe Atemleistung (etwa 0,01 Watt) für die Inhalation ausreichend, sodass auch Patienten mit Asthma und starker Obstruktion die Inhalation mit gutem Ergebnis ausführen können.

\section{Inhalations-Coach überprüft Inhalationsmanöver}

Für eine erfolgreiche Inhalationstherapie zur Behandlung von Atemwegserkrankungen ist eine Grundvoraussetzung, dass der Patient die Inhalation des verordneten Medikaments korrekt ausführt. Mit dem neuen Inhalations-Coach kann der eigentliche Inhalationsvorgang und die Qualität des Inhalationsmanövers kontrolliert werden. Bei dem Inhalations-Coach handelt es sich um ein computergestütztes Messsystem, das bei der Inhalation Atem- flüsse und inspiratorische Manöver von Patienten misst und so eine objektive Bewertung der Inhalationsqualität erleichtert. Der Inhalations-Coach kommt seit Mitte Juni 2015 zunächst in pneumologischen Praxen zum Einsatz. Praktische Tipps für die Gestaltung eines Aktionstags mit dem Inhalations-Coach und Infoflyer für Patienten runden das Angebot ab. Interessierte Ärzte können eine Nachricht an inhalations-coach@mundipharma.de senden.

\section{Friederike Klein, München und} Pressemitteilung (Mundipharma)

${ }^{1}$ Vortrag „Power requirements of pMDIs and DPIs“ im Rahmen des 20th ISAM Congress, München, Juni 2015, Veranstalter: Mundipharma Deutschland GmbH \& Co KG

${ }^{2}$ Haidl P et al. 20th ISAM Congress, Munich : Poster 238

${ }^{3}$ Flutiform $^{\circledR}$, Mundipharma Deutschland GmbH \& Co KG, Limburg

\section{Asthma bronchiale und COPD \\ Passende Wirkstärke wählen}

Den bewährten Wirkstoff Budesonid ${ }^{1}$ gibt es jetzt in 3 Wirkstärken: 0,25, 0,5 und $1 \mathrm{mg}$, für eine größtmögliche TherapieIndividualität mit effektiver Wirkung und sicherer Anwendung bei Asthma bronchiale und COPD. Es stehen 3 Packungsgrößen zu 20, 40 und 60 Ampullen zur Verfügung. In der 60er-Packung sind sie effektiv kombiniert mit dem in Zulassungsstudien bewährten Vernebler.

Das Präparat ist zur Behandlung von Asthma bronchiale zugelassen in den Fällen, bei denen Treibmittel- oder Pulverinhalatoren nicht geeignet sind. Es ist nicht zur Behandlung akuter Asthmaanfälle indiziert.

Außedem ist es zugelassen zur Behandlung von akuten Exazerbationen bei chronisch obstruktiver Bronchitis (COPD) in den Fällen, in denen die Vernebelung von Budesonid angebracht ist.

Nach einer Pressemitteilung

(Pari)

${ }^{1}$ Buparid $^{\circledR}$, Pari GmbH, Starnberg 